\title{
Reduced Variability of Endurance Time in New Protocols for Exercise Tests in COPD
}

This article was published in the following Dove Press journal:

International Journal of Chronic Obstructive Pulmonary Disease

\author{
Ellen Tufvesson (1D) \\ Finn Radner (D) \\ Georgia Papapostolou' \\ Linnea Jarenbäck (1D' \\ Saga Jönsson' \\ UIf Nihlén' \\ Jaro Ankerst' \\ Alf Tunsäter $\mathbb{D}^{\prime}$ \\ Stefan Peterson ${ }^{2}$ \\ Leif Bjermer (iD) \\ Göran Eriksson (DD' \\ 'Department of Clinical Sciences Lund, \\ Respiratory Medicine and Allergology, \\ Lund University, Skane University \\ Hospital, Lund, Sweden; ${ }^{2}$ Regional \\ Cancer Centre South, Region Skane, \\ Lund, Sweden
}

Correspondence: Ellen Tufvesson Department of Clinical Sciences Lund, Respiratory Medicine and Allergology, Lund University, Skane University Hospital, Lund 22185, Sweden Tel +46736401916

Email ellen.tufvesson@med.lu.se
Purpose: For exercise testing of COPD patients, a standard endurance test (ET) with constant workload is recommended. The test suffers from large inter-individual variability and need for large sample sizes in order to evaluate treatment effects.

Methods: A new protocol for ET in COPD was designed. In contrast to the standard ET, the new ET involved an increasing workload in order to reduce the standard deviation of endurance time. Two new ETs were compared with the standard ET. In Study A, the new ET started at $75 \%$ of the patient's maximum workload $\left(\mathrm{W}_{\text {MAX }}\right)$ and increased stepwise with $3 \% / 2$ min until exhaustion. Study B started at $70 \%$ of $\mathrm{W}_{\text {MAX }}$ and increased linearly with $1 \%$ / $\min$.

Results: In Study A, that included 15 patients, the standard deviation and range for endurance time and work capacity were narrower for the new versus the standard ET. However, the higher mean workload at end and the low mean work capacity relative to the standard ET indicated that the stepwise increase was too aggressive. In Study B, that included 18 patients, with a modified protocol, the averages for endurance time, workload at end and work capacity were similar for new and standard ET, while the standard deviations and ranges for endurance time and work capacity were kept more narrow in the new ET. The variances for endurance time were not equal between the standard ET and the two new ETs ( $<<0.05$ for both according to Levene's test).

Conclusion: The new ET reduced the number of patients with extreme endurance times (short and long) compared to the standard test. The new test showed a significant lower variance for endurance time, which potentially can lead to fewer patients needed in comparative studies. The overall best results were observed with a low linear increase during endurance.

Keywords: COPD, exercise testing, constant endurance test, endurance time

\section{Introduction}

Response to therapy in COPD patients has been mainly assessed by symptoms, number of exacerbations and resting pulmonary function. However, prognosis and quality of life is more accurately associated with exercise (in)tolerance. ${ }^{1-6}$ There are several ways of classifying exercise tests, with only minor changes in contemporary publications in comparison to a printed version from WHO in $1971 .^{7}$ Various exercise tests can be mainly categorized based on the intensity of effort, either as a maximum or a submaximum test, or based on applying the workload as either on a single constant level throughout the test, or as continuous (linear or stepwise) increased load. ${ }^{7}$

A particular approach of cardiopulmonary exercise testing has gained popularity over the years in evaluating the effect of interventions in COPD patients, namely 
the constant work-rate exercise test. ${ }^{8}$ This is a sub-maximum constant cycle endurance test (ET) at $75 \%$ of maximum achieved workload, which in the following text will be referred to as the standard ET. ${ }^{8-12}$ Exercise time until exhaustion measured by a standard ET is a more sensitive method in detecting changes after intervention in comparison to maximum incremental tests and 6-minute walk tests. ${ }^{8}$ Despite this proven superiority, there is a limited usage in COPD of the standard ET, both in clinical trials and clinical practice, due to large intra- and interindividual variability. Exceedingly long endurance times have repeatedly been reported. In the study by Worth et $\mathrm{al}^{12}$ one patient bicycled for $30 \mathrm{~min}$, and in the study by Maltais et al, ${ }^{9} 2$ patients had endurance times of around $50 \mathrm{~min}$. Too short endurance times are also problematic for the evaluation of the study results. Ten out of 92 patients had an endurance time of less than 3 min during a standard ET in a study by Vivodtzev et al, ${ }^{11}$ and 43 of $463 \mathrm{had}$ an endurance time of less than $3.4 \mathrm{~min}$ in a study by O'Donnell et al. ${ }^{5}$ Because of the great variability, large sample sizes are needed to evaluate treatment effects in clinical trials. $^{9-11}$

Elimination of long endurance times during the standard ET will reduce the phenomenon of very long postintervention tests, requiring premature termination by the investigators and therefore invalidating interpretation of the magnitude of intervention-related endurance time change using parametric statistics. ${ }^{13-15}$ In an official statement by the ERS, ${ }^{8}$ the target endurance time of a preintervention standard ET should be between 3 and $8 \mathrm{~min}$, eg, for evaluation of rehabilitation. There are some important physiological, statistical, and practical reasons necessitating this relatively narrow range. ${ }^{1,4,14,15}$

The initial step of cardiopulmonary exercise testing involves acquiring a maximum workload $\left(\mathrm{W}_{\mathrm{MAX}}\right)$ by a maximum incremental exercise test (standard $\mathrm{W}_{\mathrm{MAX}}$ test). The existing protocols for a standard $\mathrm{W}_{\mathrm{MAX}}$ test may lead to either an underestimation or overestimation of maximum workload, leading to too long and short endurance time, respectively. In a previous study, ${ }^{16}$ a prediction algorithm for calculation of $\mathrm{W}_{\mathrm{MAX}}$ in COPD patients was developed based on bicycle exercise ET results from large industry-sponsored clinical trials. The best algorithm included anthropometrics and six lung function parameters, of which forced expiratory volume in 1 second $\left(\mathrm{FEV}_{1}\right)$ and diffusion capacity for carbon monoxide (DLCO) were the best predictors. The study also included a new $\mathrm{W}_{\text {MAX }}$ test where the participants started biking with a 3-min warm-up at $40 \%$ of their predicted $\mathrm{W}_{\text {MAX }}$, followed by an instant increase to $70 \%$ and then a linear increase in workload to achieve the predicted $\mathrm{W}_{\mathrm{MAX}}$ within $8 \mathrm{~min}$. The two tests correlated well, but the new $\mathrm{W}_{\mathrm{MAX}}$ test had benefits: continuous (not categorical) increase, total work performed was higher with less deviation and better correlation to the prediction algorithm even though the algorithm was developed from the standard $\mathrm{W}_{\text {MAX }}$ test.

Although it is difficult to specify all the contributors resulting in large variability in endurance time, it is suggested that an ET performed at a fixed proportion of maximum incremental workload capacity does not provide the same physiological stress among individuals and this approach is unlikely to result in the optimal endurance time. ${ }^{11}$

Our overall aim is to improve the ET to a level where smaller studies comparing treatments can be performed and still conclusive outcomes can be obtained. We therefore aim to reduce the variability of the ET, ie, so that too short and too long endurance times can be avoided according to recommendations of ERS. ${ }^{8,11,15}$ In this methodological study, we specifically aimed to investigate a new sub-maximum, dis-continuous ET protocol on COPD patients.

\section{Patients and Methods}

\section{Patients}

Both studies enrolled outpatients with COPD, with a postbronchodilator $\mathrm{FEV}_{1}$ of $40-80 \%$ of predicted normal, a ratio of $\mathrm{FEV}_{1}$ to forced vital capacity (FVC) of less than 0.7 and no cardiovascular co-morbidity preventing exercise testing or any other disease that could put the patients at increased risk or interfere with the study results. Subjects with a history of COPD exacerbation within 6 months prior to the study were excluded.

\section{Ethics}

The Regional Ethical Review Board in Lund, Sweden, approved the studies, which complied with the Declaration of Helsinki. Written informed consent was obtained from all patients prior to any study-related procedure.

\section{Study Design}

Studies A and B were randomized single-centre cross-over trials. Patients not fulfilling the inclusion/exclusion criteria at visit 1 were excluded. Baseline demographic and lung function data (Table 1) were used to calculate the predicted maximum workload $\left(\mathrm{W}_{\mathrm{MAX}}\right)$ in $\mathrm{W}$ for each patient as 
Table I Patient Baseline Characteristics

\begin{tabular}{|c|c|c|}
\hline & Study A & Study B \\
\hline Number of patients & 15 & 18 \\
\hline Age, years & $71 \pm 5$ & $71 \pm 5$ \\
\hline Male, n (\%) & II (73) & $10(56)$ \\
\hline Height, cm & $172 \pm 9$ & $17 \mid \pm 10$ \\
\hline Weight, kg & $77 \pm 12$ & $77 \pm 11$ \\
\hline BMI, $\mathrm{kg} / \mathrm{m}^{2}$ & $26 \pm 3$ & $26 \pm 3$ \\
\hline Previous/current smokers, n (\%) & $14(93) / /(7)$ & $17(94) / /(6)$ \\
\hline Pack years, median (range) & $30(10-68)$ & $33(10-65)$ \\
\hline $\begin{array}{l}\text { Time since diagnosis, } 2-5 \text { years } />5 \\
\text { years, } n\end{array}$ & $3 / 12$ & $4 / 14$ \\
\hline $\mathrm{FEV}_{1}, \mathrm{~L}^{\mathrm{a}}$ & $1.80 \pm 0.44$ & $1.76 \pm 0.37$ \\
\hline $\mathrm{FEV}_{\mathrm{l}}, \%$ of predicted ${ }^{\mathrm{a}}$ & $60.3 \pm 8.6$ & $64.5 \pm 9.7$ \\
\hline FVC, $L^{\mathrm{a}}$ & $3.62 \pm 1.07$ & $3.39 \pm 0.84$ \\
\hline $\mathrm{FEV}_{\mathrm{l}} / \mathrm{FVC}^{\mathrm{a}}$ & $0.51 \pm 0.09$ & $0.53 \pm 0.10$ \\
\hline Total CCQ score, median (range) & $1.0(0.2-2.2)$ & $0.9(0-3.0)$ \\
\hline $\begin{array}{l}\text { CCQ symptom score, median } \\
\text { (range) }\end{array}$ & $1.3(0.3-2.3)$ & $1.3(0-3.8)$ \\
\hline CCQ mental score, median (range) & $0.0(0-2.0)$ & $0.5(0-2.5)$ \\
\hline CCQ function score, median (range) & $1.0(0-2.8)$ & $0.5(0-3.0)$ \\
\hline \multicolumn{3}{|l|}{ Medications used at study entry, $\mathrm{n}(\%)$ : } \\
\hline Any ICS & $9(60)$ & $5(28)$ \\
\hline Any LABA & $8(53)$ & $9(50)$ \\
\hline Any LAMA & $14(93)$ & 16 (89) \\
\hline
\end{tabular}

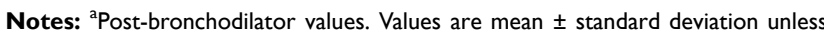
otherwise stated.

Abbreviations: BMI, body-mass index; CCQ, Clinical COPD Questionnaire; FEV forced expiratory volume in I second; FVC, forced vital capacity; ICS, inhaled corticosteroid; LABA, long-acting $\beta 2$-receptor agonist; LAMA, long-acting muscarinic receptor antagonist.

previously described. ${ }^{16}$ At all visits, patients received a fast-acting bronchodilator, ie, all measurements and exercise tests are post-bronchodilator administration. Before all exercise tests, patients filled out the Clinical COPD Questionnaire (CCQ) and underwent a physical examination including vital signs, ECG and dynamic spirometry. A physician assessed the patient's clinical status and approved or disapproved further participation. All exercise tests involved continuous measurement of $\mathrm{VO}_{2}, \mathrm{VCO}_{2}$, respiratory rate and heart rate. Borg dyspnoea score and Borg leg discomfort score ${ }^{17}$ were reported every second minute, and measurements of ECG and blood pressure were performed before and after exercise. Both studies included measurements of $\mathrm{W}_{\mathrm{MAX}}$ from the standard maximum test (Study A) and/or the new maximum test (Study $\mathrm{A}^{16}$ and Study B). The new maximum test included an initial phase of pedalling for $3 \mathrm{~min}$ at $40 \%$ (Study A) or $30 \%$ (Study B) of the predicted $\mathrm{W}_{\mathrm{MAX}}$, followed by an instant increase to $75 \%$ (Study A) or $70 \%$ (Study B), followed by a stepwise (Study A) or linear (Study B) increase in workload until exhaustion.

\section{Study A}

Study A, consisted of a screening visit (visit 1), not including any exercise tests, followed by 4 visits with exercise testing (Figure 1; Study A). At visit 2, enrolled patients were randomized to either the standard $\mathrm{W}_{\text {MAX }}$ test or the new $\mathrm{W}_{\mathrm{MAX}}$ test as previously reported, ${ }^{16}$ in order to obtain the measured $\mathrm{W}_{\mathrm{MAX}}$ to be used as the basis for the starting values for each ET.

Patients randomized to the standard $\mathrm{W}_{\mathrm{MAX}}$ test (performed at visit 2), performed a standard ET (at visit 3) at $75 \%$ of measured $\mathrm{W}_{\text {MAX }}$ until exhaustion was reached in accordance with the standard methodology. Patients randomized to the new $\mathrm{W}_{\mathrm{MAX}}$ test (performed at visit 2) instead performed a new ET ( $\mathrm{ET}_{\mathrm{A}}$; at visit 3). After a 3minute period of low workload cycling ( $40 \%$ of the measured $\mathrm{W}_{\mathrm{MAX}}$ ), the load was increased to $75 \%$ and then stepwise increased with $3 \%$ every second minute until the patient stopped due to exhaustion (Figure 2; Study A). At visits 4 and 5 the patients were crossed over to complete the opposite tests (Figure 1; Study A).

After completion of Study A, the new $\mathrm{ET}_{\mathrm{A}}$ was modified to aim for a decrease in the higher mean workload and lower work capacity compared to the standard ET. A number of modifications (decreased start workload and less escalation intensity) were implemented to achieve a less intense escalation and for subsequent use in Study B.

\section{Study B}

Study B consisted of a screening visit including the new $\mathrm{W}_{\text {MAX }}$ test (visit 1), followed by two visits with ETs (Figure 1: Study B). At visit 1, eligible patients performed the new $\mathrm{W}_{\mathrm{MAX}}$ test. At visit 2, enrolled patients were randomized to either the standard ET or the new modified ET $\left(\mathrm{ET}_{\mathrm{B}}\right)$. The standard ET was the same as in Study A, albeit with a constant workload of $75 \%$ of measured $\mathrm{W}_{\text {MAX }}$ obtained from the new $\mathrm{W}_{\text {MAX }}$ test instead of the standard $\mathrm{W}_{\text {MAX }}$ test as in Study A.

The new modified $\mathrm{ET}_{\mathrm{B}}$ started with an initial workload for 3 minutes at $30 \%$ of measured $\mathrm{W}_{\text {MAX }}$ obtained from the new $\mathrm{W}_{\text {MAX }}$ test. Then, the workload was set to $70 \%$, followed by a linear increase of $1.0 \%$-units per min until the patient reached his/her maximum workload and stopped due to exhaustion (Figure 2: Study B). At visit 3 the patients were crossed-over to the other test (Figure 1: Study B). 


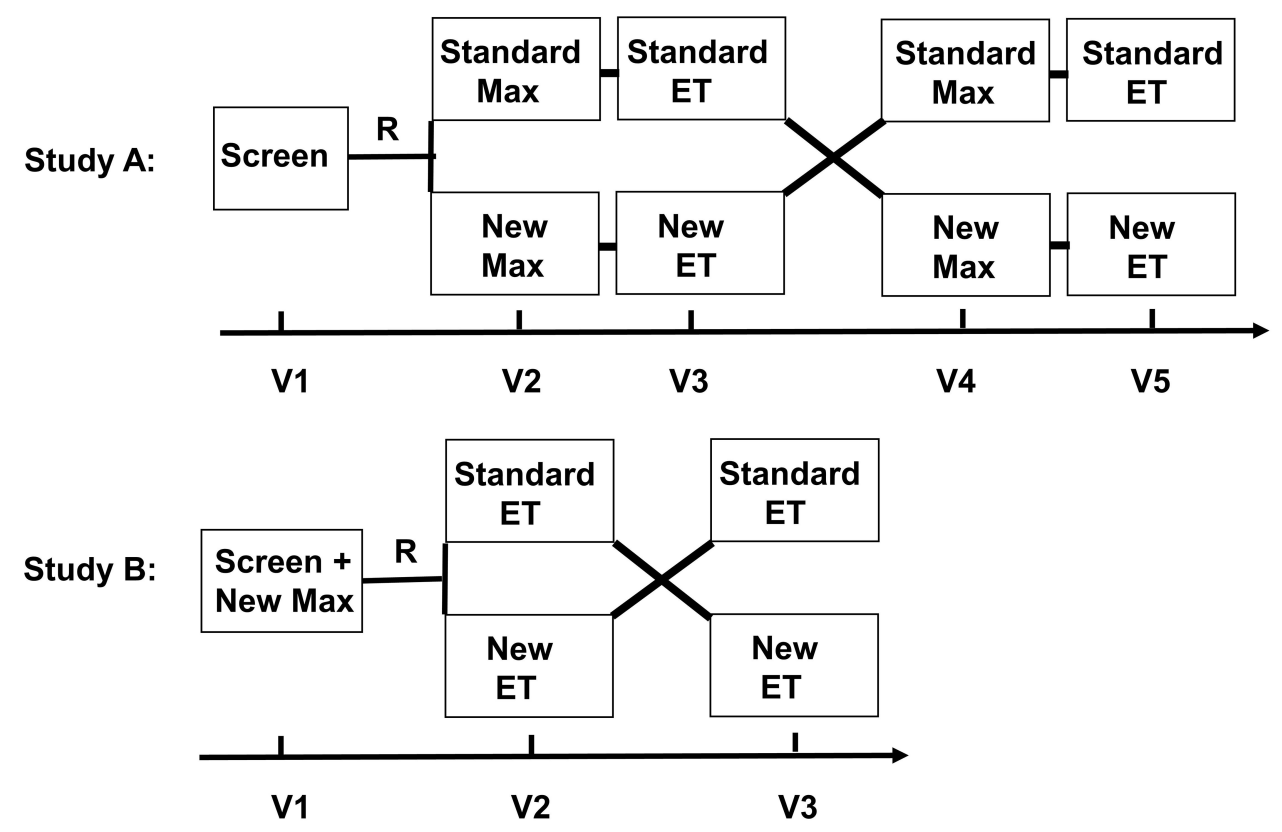

Figure I Schematic picture of study design for standard and new tests in Study A and Study B. Abbreviations: $E T$, endurance test; Max, $\mathrm{W}_{\text {MAX }}$ test; $R$, randomisation; Screen, screening visit; $V$, visit.

\section{Study Equipment}

Patients performed (MasterScreen, Erich Jaeger $\mathrm{GmbH}$, Würzburg, Germany) and ergospirometry (Oxycon Pro ${ }^{\mathrm{TM}}$, Erich Jaeger GmbH, Würzburg, Germany).

\section{Outcome Measures}

In both studies, the primary outcome measure was the nonequivalence of the variance of the endurance times for the standard $\mathrm{ET}$ and the new $\mathrm{ET}_{\mathrm{A}} / \mathrm{ET}_{\mathrm{B}}$, obtained from Levene's test.

The secondary outcome variable was a descriptive comparison between the standard ET and the new $\mathrm{ET}_{\mathrm{A}} /$ $\mathrm{ET}_{\mathrm{B}}$ for the range of the endurance times. The work capacity (kWs) was compared between the standard ET and the new $\mathrm{ET}_{\mathrm{A}} / \mathrm{ET}_{\mathrm{B}}$ in the same way as endurance time.

Other outcome variables such as workload at end (W), Borg scale results, oxygen kinetics measurements and reasons for stopping exercise were descriptively compared between the standard and new $\mathrm{ET}_{\mathrm{A}} / \mathrm{ET}_{\mathrm{B}}$.

\section{Statistical Analyses}

We designed the studies to include 15-20 patients in order to obtain enough data to compare the new $\mathrm{ET}_{\mathrm{A}} / \mathrm{ET}_{\mathrm{B}}$ with the standard ET, but no formal power calculations were performed. Levene's test for equal variance was performed in IBM SPSS (v 25 for Windows). Further comparisons between the two tests were performed using descriptive statistics and boxplots using Microsoft Excel for Office 365. Demographics and patient data were expressed as mean \pm standard deviation unless otherwise stated. Work capacity in kWs was calculated as area-under-curve from workload (W) x time (s) (Figure 2).

\section{Results}

\section{Patient Populations Study A}

In total, 19 patients were enrolled between July and October 2015. Among them, three patients were excluded due to $\mathrm{FEV}_{1} \geq 80 \%$ predicted, and one patient due to significant cardiovascular co-morbidity. The remaining 15 patients were included in the study and performed all study procedures. They had a mean age of 71 years, a mean $\mathrm{FEV}_{1}$ of $60 \%$ of predicted normal, and an $\mathrm{FEV}_{1} / \mathrm{FVC}$ ratio of 0.51 . Further patient baseline characteristics are presented in Table 1.

\section{Study B}

In total, 30 patients were enrolled between July 2016 and February 2018. Six patients were excluded due to $\mathrm{FEV}_{1} \geq$ $80 \%$ predicted, two patients due to significant cardiovascular co-morbidity and one patient due to other relevant co-morbidity (inguinal hernia). Twenty-one patients were included in the study and performed the new $\mathrm{W}_{\text {MAX }}$ test at visit 1. Among these 21 patients, one left the study between visit 1 and 2, and two patients completed visit 

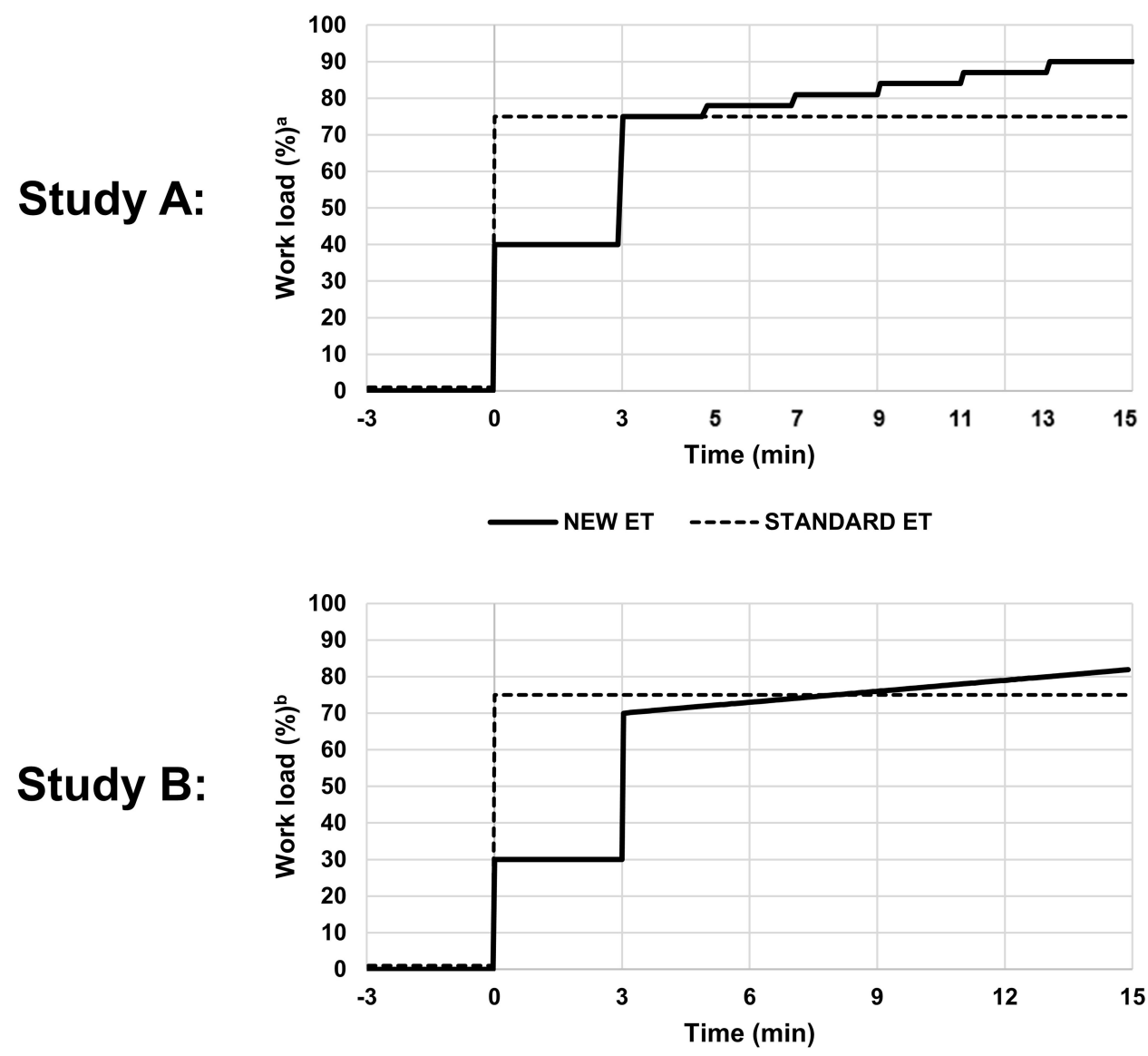

NEW ET -----STANDARD ET

Figure 2 Schematic design of the endurance tests in Study A and Study B.

Notes: ${ }^{a}$ Workload in \% of maximum value obtained from the new maximum test (in the new endurance test) or from the standard maximum test (in the standard endurance test). ${ }^{b}$ Workload in \% of maximum value obtained from the new maximum test. Baseline ( -3 to 0 min) and recovery phase are similar in the new endurance test and the standard endurance test.

Abbreviations: ET, endurance test; min, minutes.

2, but then left the study, leaving 18 patients who completed all visits. These patients had a mean age of 70 years, a mean $\mathrm{FEV}_{1}$ of $64 \%$ of predicted normal and an $\mathrm{FEV}_{1} / \mathrm{FVC}$ ratio of 0.55 . Further demographics and baseline characteristics are provided in Table 1.

Results from the $\mathrm{W}_{\text {MAX }}$ tests in Studies A and B are shown in Table S1.

\section{Comparison of the New and the Standard Endurance Test in Study A}

The patients tested with the new $\mathrm{ET}_{\mathrm{A}}$ compared to the standard ET reached a numerically higher measured workload at the end of the test ( 89 versus $80 \mathrm{~W}$ ). The endurance time was similar between new and standard $\mathrm{ET}$, albeit the new $\mathrm{ET}_{\mathrm{A}}$ included 3 minutes on a low workload $(40 \%)$.
The new $\mathrm{ET}_{\mathrm{A}}$ had a narrower range of the endurance time (6.2-14.7 versus 3.3-20.9), showing that fewer patients had a very short or very long endurance time on the new $\mathrm{ET}_{\mathrm{A}}$ compared to the standard ET. Two patients had an endurance time $\geq 20$ min and two patients had an endurance time below 5 min during the standard ET, compared to 0 and 0 , respectively, with the new $\mathrm{ET}_{\mathrm{A}}$ (Tables $\mathrm{S} 2$ and $\mathrm{S} 3$ ).

The standard deviation in \% of mean endurance time for the new $\mathrm{ET}_{\mathrm{A}}$ was less than half that of the standard $\mathrm{ET}$ ( $24 \%$ versus $51 \%$ ). Levene's test showed that the variances for the endurance time of the two tests were not equal; $\mathrm{F}(1.28)=12.96 ; \mathrm{p}=0.001$.

The new $\mathrm{ET}_{\mathrm{A}}$ used less work capacity (46 versus 56 kWs; Table 2, Figure 3) and a narrower range and a lower standard deviation than the standard ET was demonstrated. Levene's test did not show a statistically significant difference in variance $(\mathrm{p}=0.066)$. 
Table 2 Results from the Endurance Tests in Study A and Study B

\begin{tabular}{|c|c|c|c|c|}
\hline & \multicolumn{2}{|l|}{ Study A } & \multicolumn{2}{|l|}{ Study B } \\
\hline & Standard ET & New $\mathbf{E T}_{\mathbf{A}}$ & Standard ET & New $\mathrm{ET}_{\mathrm{E}}$ \\
\hline \multicolumn{5}{|l|}{ Workload } \\
\hline Workload at start, W & $80 \pm 17$ & $42 \pm 9$ & $75 \pm 24$ & $30 \pm 9$ \\
\hline Workload at $3 \mathrm{~min}, \mathrm{~W}$ & $80 \pm 17$ & $79 \pm 17$ & $75 \pm 24$ & $70 \pm 23$ \\
\hline Workload at end, W & $80 \pm 17$ & $89 \pm 23$ & $75 \pm 24$ & $78 \pm 27$ \\
\hline SD in \% of Work load at end & $21 \%$ & $26 \%$ & $32 \%$ & $34 \%$ \\
\hline Range of Work load at end, W & $53-113$ & $68-153$ & $33-137$ & $33-146$ \\
\hline \multicolumn{5}{|l|}{ Endurance time } \\
\hline Endurance time, $\min$ & $10.9 \pm 5.6$ & $10.3 \pm 20.5$ & $9.9 \pm 5.6$ & $10.7 \pm 3.0$ \\
\hline SD in \% of endurance time & $51 \%$ & $24 \%$ & $57 \%$ & $28 \%$ \\
\hline Median endurance time, $\min$ & 12.1 & 10.7 & 7.9 & 9.4 \\
\hline Range of endurance time, min & $3.3-20.9$ & $6.2-14.7$ & $2.8-21.7$ & $7.4-16.3$ \\
\hline Levene's test: Standard vs New & \multicolumn{2}{|c|}{$F(1.28)=12.96 ; p=0.001$} & \multicolumn{2}{|c|}{$F(I .34)=4.5 I ; p=0.04 I$} \\
\hline \multicolumn{5}{|l|}{ Work performed } \\
\hline Work capacity, kWs & $57 \pm 38$ & $46 \pm 21$ & $46 \pm 35$ & $42 \pm 25$ \\
\hline SD in \% of work performed & $69 \%$ & $48 \%$ & $76 \%$ & $59 \%$ \\
\hline Median Work capacity, kWs & 54 & 39 & 40 & 34 \\
\hline Range of Work capacity, kWs & $12-142$ & $18-86$ & $9-143$ & $12-97$ \\
\hline Levene's test: Standard vs New & \multicolumn{2}{|c|}{$F(1.28)=3.66 ; p=0.066$} & \multicolumn{2}{|c|}{$F(1.34)=0.168 ; p=0.46$} \\
\hline
\end{tabular}

Notes: Values are mean \pm standard deviation unless otherwise specified.

Abbreviation: vs, versus.

Five patients stopped the new $\mathrm{ET}_{\mathrm{A}}$ due to leg discomfort compared to one patient at the standard ET, while six patients at each test stopped due to dyspnea. Borg scale scores at end and peak $\mathrm{VO}_{2} \mathrm{VCO}_{2}$, minute ventilation and respiratory ratevalues were similar between the tests (Table 3 ).

The higher workload at the end, and the lower work capacity relative to the standard ET, indicated that the step up of workload was too aggressive. Also, more patients stopped the $\mathrm{ET}$ due to leg discomfort during the new $\mathrm{ET}_{\mathrm{A}}$. A less powerful step up was therefore chosen for Study B, as described in the 'Methods' section.

\section{Comparison of the New and the Standard Endurance Test in Study B}

The patients tested with the new $\mathrm{ET}_{\mathrm{B}}$ compared to the standard ET reached slightly higher workload at the end of exercise (78 versus $75 \mathrm{~W}$ ). The mean endurance time was similar to that in study A comparing the new and standard ET, albeit new $\mathrm{ET}_{\mathrm{B}}$ included 3 minutes on a low workload (30\%).

The new $\mathrm{ET}_{\mathrm{B}}$ had a narrower range of the endurance time (7.4-16.3 versus $2.8-21.7)$ compared to the standard
ET, showing that fewer patients bicycled very short or very long (Figure 3). Two patients had an endurance time $\geq 20$ min during the standard ET and 3 patients had

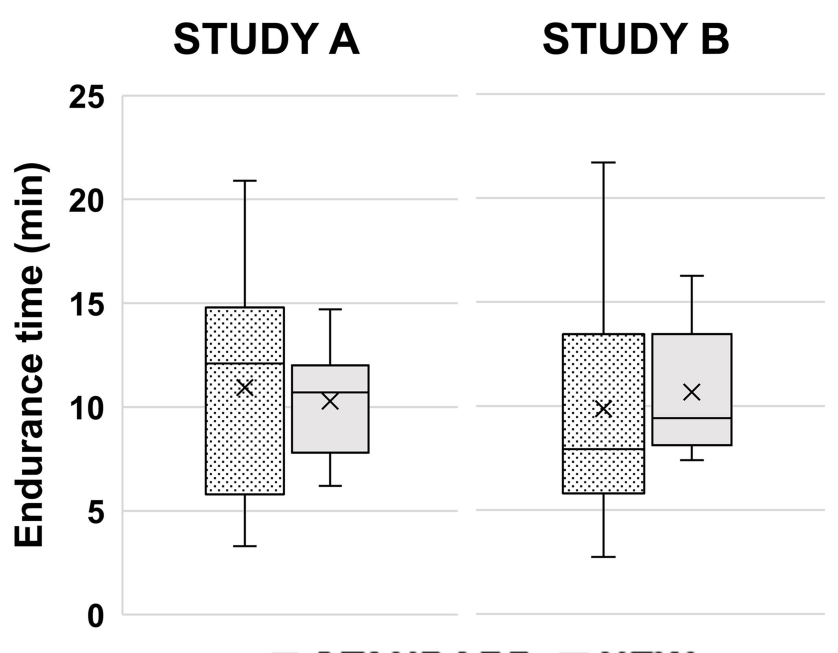

STANDARD $\square$ NEW

Figure 3 Box plots of endurance times in minutes $(\mathrm{min})$ in the standard and new endurance tests in Study A and Study B.

Notes: Boxes show 25-75 percentiles, whiskers show minimum and maximum values, lines through the boxes show median and crosses show mean values. 


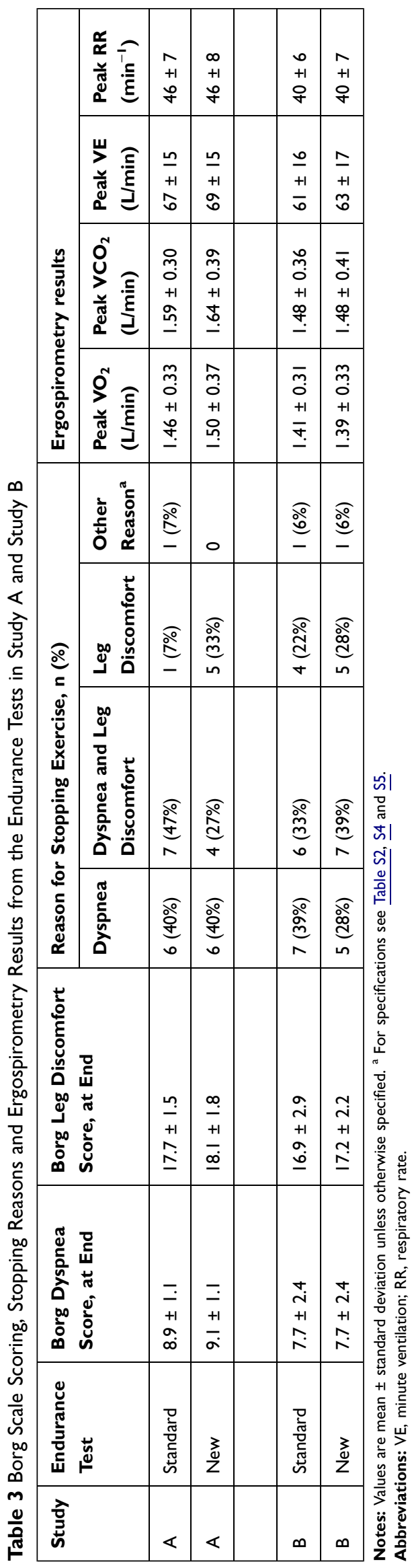

an endurance time below 5 min compared to 0 and 0 , respectively, with the new $\mathrm{ET}_{\mathrm{B}}$ (Tables $\mathrm{S} 4$ and $\underline{\mathrm{S} 5}$ ). The standard deviation in $\%$ of mean endurance time for the new $\mathrm{ET}_{\mathrm{B}}$ was less than half that of the standard ET ( 28 versus $57 \%$ ). Levene's test showed that the variances for the endurance time of the two tests were not equal $(\mathrm{F}$ $(1.34)=4.51 ; \mathrm{p}=0.041)$.

The new $\mathrm{ET}_{\mathrm{B}}$ used slightly less work capacity (42 versus $46 \mathrm{kWs}$; Table 2, Figure 3) and demonstrated a narrower range and a lower standard deviation in comparison to the standard ET, but Levene's test did not demonstrate a significant difference in variance $(\mathrm{p}=0.46)$.

Table 3 presents Borg scale results and reasons for stopping the ET. Five patients stopped the new $\mathrm{ET}_{\mathrm{B}}$ due to leg discomfort compared to four for the standard ET, while five and seven patients, respectively, stopped due to dyspnea. Borg scale scores at end and peak $\mathrm{VO}_{2}, \mathrm{VCO}_{2}$, minute ventilation and respiratory rate-values were similar between the tests (Table 3).

\section{Comparison of the Two New ET Tests ( $E T_{A}$ and $\left.E_{B}\right)$ Using the Standard ET as Reference}

The mean endurance time was similar across all three ET tests. However, the standard deviation for endurance time (tested as difference in variance using Levene's test) was statistically significantly lower for both the two new ET tests as compared to the standard ET. In addition, the narrower range of endurance time in both studies resulted in similarly low numbers of patients cycling very short and very long compared to the standard ET.

However, compared to the standard ET the mean workload at end differed numerically less in $\mathrm{ET}_{\mathrm{B}}$ ( 78 versus 74 $\mathrm{W}=4 \%$ ) than in $\mathrm{ET}_{\mathrm{A}}$ ( 89 versus $80 \mathrm{~W}=11 \%$ ). In addition, the mean work capacity also differed less in $\mathrm{ET}_{\mathrm{B}}(42$ versus $46=-9 \%$ ) than in $\mathrm{ET}_{\mathrm{A}}$ (46 versus $56=-18 \%$ ) compared to the standard ET. The finding of a lowered standard deviation for work capacity was similar in the two new ET tests, although the test of variance did not reach significance $\left(\mathrm{ET}_{\mathrm{A}}: 48\right.$ versus $69 \%, \mathrm{p}=0.066$ and $\mathrm{ET}_{\mathrm{B}}: 59$ versus $76 \%, p=0.46$ ). In addition, the narrower range of work capacity was seen for both new ETs compared to the standard ET.

Borg scale scores at end of dyspnea and leg discomfort, as well as oxygen kinetic data at peak, were similar across all three ETs. The number of patients that stopped due to dyspnea was also similar in all studies. However, the 
number of patients who stopped due to leg discomfort was larger in $\mathrm{ET}_{\mathrm{A}}$ compared to the standard ET (5 (33\%) versus 1 patient $(7 \%)$ ), but in study $\mathrm{B}$, the number of patients who stopped due to leg discomfort was similar to the standard ET (5 (28\%) versus $4(22 \%))$.

Taking all the above results into consideration, $\mathrm{ET}_{\mathrm{B}}$ was preferred over $\mathrm{ET}_{\mathrm{A}}$ in relation to the standard ET.

\section{Discussion}

Our objective was to develop a new sub-maximum ET for COPD patients, with only a low number of patients required for making a clinically relevant evaluation of treatment effects. It was designed to achieve both a reasonably narrow range of endurance time (avoiding too short and too long endurance times), and a variance well below that of the standard ET. The results of this study suggest that a new sub-maximum ET with increasing workload is capable to narrow the range of endurance time and reduce its variance compared to the standard constant workload ET.

In Study A, the sub-maximum ET started at $40 \%$ of $\mathrm{W}_{\text {MAX }}$ from the preceding $\mathrm{W}_{\text {MAX }}$ test, increased to $75 \%$ after three minutes and thereafter the workload increased stepwise with $3 \% / 2 \mathrm{~min}$ until exhaustion. This protocol significantly reduced standard deviation with $50 \%$ (ie, the variance differed significantly) and reduced the range for endurance time compared to the standard ET. Also, the work capacity had lower standard deviation (30\%, NS). The mean endurance times were similar between the two tests, but workload at the end increased $(+11 \%)$ and work capacity decreased $(-18 \%)$ with the new protocol compared to the standard ET. Our conclusion was that the new test fulfilled our objectives, but the stepwise escalation resulted in a workload at end close to $90 \%$ of $\mathrm{W}_{\text {MAX }}$. In Study B we therefore reduced the workload to $30 \%$ during the initial three minutes, followed by an instant increase to $70 \%$, ie, lower than in the first study and also lower than the $75 \%$ in the standard ET. Thereafter, the workload was increased with $1 \%$ per minute in a linear fashion until exhaustion was reached. This protocol also resulted in a significantly reduced standard deviation with $50 \%$ (ie, the variance differed significantly) and reduced the range for endurance time. Mean workload at the end and mean work capacity came closer to the standard ET.

Both protocols fulfilled the objective of lowering the variance and making the range of the endurance time narrower. However, the sub-maximum linear new test protocol in Study B was superior. Firstly, because many mean values of outcomes were similar to the standard ET, including similar of patients that stopped due to leg fatigue. Secondly, because the lower starting loads of $30 \%$ and $70 \%$ would benefit patients with a preceding low $\mathrm{W}_{\text {MAX }}$ test and where $\mathrm{W}_{\mathrm{MAX}}$ was over-estimated, ie, $70 \%$ will be more forgiving than a $75 \%$ start. Thirdly, because the linear $1 \% /$ min escalation meant a slower continuous increase compared to a higher stepwise (discontinuous) increase, while keeping endurance phase well below $20 \mathrm{~min}$.

A constant work rate at $75 \%$ is most commonly used in exercise testing in COPD but also a constant work rate at $85 \%$ has been used. The constant work rate test has been shown to be reliable and reproducible but there are some limitations. As the relation between endurance time and work capacity is not linear, a fixed constant percentage of the ET will be dependent on whether the patients are below or above the time versus workload curve and the accuracy of the incremental maximum test. Both can lead to extremely short and long endurance times. In Study B, the range of endurance time was 2.8 to 21.7 minutes for the standard ET. In two studies ${ }^{5,11}$ (one including pooled data from two separate studies ${ }^{5}$ ), the ranges for the standard ET were 1-25 minutes and it has been reported that COPD patients have kept on bicycling for $30 \mathrm{~min}^{12}$ and 50 min, ${ }^{9}$ respectively. The shorter endurance times $(<2 \mathrm{~min})$ can be unreliable and difficult for recordings, while longer times ( $>20 \mathrm{~min})$ will lead to stopping independent of work capacity (investigator decision, boredom, seat pain, exhaustion of the muscles, etc.). ${ }^{1,4,8}$

Puente-Maestu et $\mathrm{al}^{8}$ recommended a target of 3-8 min for the pre-intervention standard ET at $75 \%$ to achieve a narrower and common baseline in rehabilitation studies, which can reduce the number of patients needed to show effect. ${ }^{8,14,15}$ Fifty-seven percent of COPD patients $(25 \%$ $<3 \mathrm{~min}$ and $18 \%>8 \mathrm{~min}$ ) was within this range in the two studies described above. ${ }^{5,11}$ A solution to increase the number of patients with reasonable endurance times could be to redo the ET at 5\% lower or higher dependent on the endurance time being low or high. The latter could lead to an additional $30 \%$ of the patients within the set range. ${ }^{5,11,18}$ This solution will increase laboratory time and cost, but it is also less feasible in a cross-over study to investigate different pharmaceuticals.

Our research question was: Why should a sub-maximum ET be conducted at constant workload, given all data showing the skewness of the constant load and the knowledge that the endurance time and the work capacity 
is not linear. We found one publication where the workload was increased during the ET. ${ }^{10}$ In their protocol, it was stated that patients reaching 15 minutes at $75 \%$ of $\mathrm{W}_{\text {MAX }}$ had the workload increased to $90 \%$. In Study A, we introduced a step-wise escalation which made the range of the endurance narrower with some patients reaching $90 \%$ of $\mathrm{W}_{\text {MAX }}$. In Study B, as explained above, the strategy was somewhat changed, partly to address patients with short endurance time, ie, to start the escalation phase at $70 \%$. With a linear increase of workload, it will take 5, 10 and $15 \mathrm{~min}$ on the escalation phase to reach $75 \%, 80 \%$ and $85 \%$ of $\mathrm{W}_{\mathrm{MAX}}$, respectively. Out of 18 patients, the shortest and longest time on this escalation phase were 4.4 and 13.7 minutes, compared to $2.8-21.7$ for the standard test. This protocol is adapted to reduce the number of outliers, who probably do not represent specific COPD patients but rather under- or over-estimation of the preceding incremental maximum test. ${ }^{8}$

The inclusion and exclusion criteria in the two studies were standard, eg, $\mathrm{FEV}_{1} 40-80 \%, \mathrm{FEV}_{1} / \mathrm{FVC}$ ratio $<70 \%$, no exacerbations the preceding 6 weeks and no cardiovascular comorbidities preventing the test. There were no further selection criteria to recruit patients more prone to response in treatment intervention studies, such as FRC $>120 \% \%^{9,10,12,19}$ and reversibility of $\mathrm{IC}^{19}$ used in other studies.

The initial 3-minute phase at a low workload (30-40\% of $\left.\mathrm{W}_{\mathrm{MAX}}\right)$ was added to investigate oxygen kinetics $\left(\mathrm{VO}_{2}\right.$ and $\mathrm{VCO}_{2}$ ) in order to add information about endurance time or work capacity. This initial phase can be deleted, especially if no additional information is achieved. However, patients have reported that this stepwise start is preferred in contrast to a direct start at $75 \%$.

Some limitations of our study could be further discussed. Both studies had a small number of participants and the studies are mainly descriptive. Neither study had a formal power calculation. However, the results for the new ET in the two studies were consistent versus the standard test and met the objectives. Our methodology could also be criticised for not being a true constantworkload test since the workload is increased during the endurance phase. The question then arises of why this test should be constant and whether the patients notice the slow linear increase $(1 \% / \mathrm{min})$. Another limitation was that the standard ET was terminated by the investigator after $20 \mathrm{~min}$ for two patients, due to protocol programming up to $20 \mathrm{~min}$ only. This pre-termination resulted in a lower standard deviation than would have been collected without the pre-termination for the standard ET. The presented data including the limitations render a validation study evaluating the benefit of reduced standard variation in a double-blind comparative study.

\section{Conclusion}

In summary, a new protocol for exercise testing in COPD has been developed. This new ET was shown to have less variance and a narrower range for endurance time and work capacity, as compared to the standard ET, and hence a reduced number of patients bicycling with extreme endurance times (both short and long). This can potentially lead to fewer patients needed in comparative studies. A sub-maximum ET with flat linear escalation, starting at $70 \%$ of $\mathrm{W}_{\mathrm{MAX}}$, seemed to provide the best overall results and will be selected for our future studies of endurance time in response to bronchodilators.

\section{Acknowledgments}

We thank the study nurses at the Department of Respiratory Medicine and Allergology, University Hospital, Lund, Sweden for their assistance during the studies.

\section{Funding}

This work was supported by the Swedish Heart and Lung foundation.

\section{Disclosure}

The authors report no conflicts of interest in this work.

\section{References}

1. American Thoracic S. American College of Chest P. ATS/ACCP statement on cardiopulmonary exercise testing. Am J Respir Crit Care Med. 2003;167(2):211-277.

2. Martinez FJ, Foster G, Curtis JL, et al. Predictors of mortality in patients with emphysema and severe airflow obstruction. Am J Respir Crit Care Med. 2006;173(12):1326-1334. doi:10.1164/ rccm.200510-1677OC

3. Oga T, Nishimura K, Tsukino M, Sato S, Hajiro T. Analysis of the factors related to mortality in chronic obstructive pulmonary disease: role of exercise capacity and health status. Am J Respir Crit Care Med. 2003;167(4):544-549. doi:10.1164/rccm.200206-583OC

4. Palange P, Ward SA, Carlsen KH, et al. Recommendations on the use of exercise testing in clinical practice. Eur Respir J. 2007;29(1):185209. doi:10.1183/09031936.00046906

5. O'Donnell DE, Travers J, Webb KA, et al. Reliability of ventilatory parameters during cycle ergometry in multicentre trials in COPD. Eur Respir J. 2009;34(4):866-874. doi:10.1183/09031936.00168708

6. Radtke T, Crook S, Kaltsakas G, et al. ERS statement on standardisation of cardiopulmonary exercise testing in chronic lung diseases. Eur Respir Rev. 2019;28:154. 
7. World Health O.; Andersen KL, Shephard RJ, Denolin H, Varnauskas E, Masironi R. Fundamentals of Exercise Testing. Geneva: World Health Organization; 1971.

8. Puente-Maestu L, Palange P, Casaburi R, et al. Use of exercise testing in the evaluation of interventional efficacy: an official ERS statement. Eur Respir J. 2016;47(2):429-460. doi:10.1183/13993003.007452015

9. Maltais F, Hamilton A, Marciniuk D, et al. Improvements in symptom-limited exercise performance over $8 \mathrm{~h}$ with once-daily tiotropium in patients with COPD. Chest. 2005;128(3):1168-1178. doi:10.1378/chest.128.3.1168

10. Magnussen H, Paggiaro P, Schmidt H, Kesten S, Metzdorf N, Maltais F. Effect of combination treatment on lung volumes and exercise endurance time in COPD. Respir Med. 2012;106(10):1413-1420.

11. Vivodtzev I, Gagnon P, Pepin V, et al. Physiological correlates of endurance time variability during constant-workrate cycling exercise in patients with COPD. PLoS One. 2011;6(2):e17007. doi:10.1371/ journal.pone. 0017007

12. Worth H, Forster K, Eriksson G, Nihlen U, Peterson S, Magnussen H. Budesonide added to formoterol contributes to improved exercise tolerance in patients with COPD. Respir Med. 2010;104(10):14501459. doi:10.1016/j.rmed.2010.07.006

13. Puente-Maestu L, SantaCruz A, Vargas T, Martinez-Abad Y, Whipp BJ. Effects of training on the tolerance to high-intensity exercise in patients with severe COPD. Respiration. 2003;70(4):367-370. doi: $10.1159 / 000072899$
14. Whipp BJ, Ward SA. Quantifying intervention-related improvements in exercise tolerance. Eur Respir J. 2009;33(6):1254-1260. doi: $10.1183 / 09031936.00110108$

15. van der Vaart H, Murgatroyd SR, Rossiter HB, Chen C, Casaburi R, Porszasz J. Selecting constant work rates for endurance testing in COPD: the role of the power-duration relationship. COPD. 2014;11 (3):267-276.

16. Eriksson G, Radner F, Peterson S, et al. A new maximal bicycle test using a prediction algorithm developed from four large COPD studies. Eur Clin Respir J. 2020;7(1):1692645. doi:10.1080/ 20018525.2019.1692645

17. Borg GA. Psychophysical bases of perceived exertion. Med Sci Sports Exerc. 1982;14(5):377-381. doi:10.1249/00005768-1982050 00-00012

18. Andrianopoulos V, Wagers SS, Groenen MT, et al. Characteristics and determinants of endurance cycle ergometry and six-minute walk distance in patients with COPD. BMC Pulm Med. 2014;14:97. doi:10.1186/1471-2466-14-97

19. Berton DC, Marques RD, Palmer B, O'Donnell DE, Neder JA. Effects of lung deflation induced by tiotropium/olodaterol on the cardiocirculatory responses to exertion in COPD. Respir Med. 2019;157:59-68. doi:10.1016/j.rmed.2019.09.006

\section{Publish your work in this journal}

The International Journal of COPD is an international, peer-reviewed journal of therapeutics and pharmacology focusing on concise rapid reporting of clinical studies and reviews in COPD. Special focus is given to the pathophysiological processes underlying the disease, intervention programs, patient focused education, and self management protocols. This journal is indexed on PubMed Central, MedLine and CAS. The manuscript management system is completely online and includes a very quick and fair peer-review system, which is all easy to use. Visit http://www.dovepress.com/testimonials.php to read real quotes from published authors. 